ARTICLE

\title{
Assessment of the Radiation Field from Withdrawal of the Internal Components in a System Integrated Reactor
}

\author{
Siwan $\mathrm{NOH}^{1}$, Hyungjoon $\mathrm{YU}^{1}$, Kyomin $\mathrm{LEE}^{1}$, Songjae $\mathrm{YOO}^{2}$ and Jaiki LEE${ }^{*}$ \\ ${ }^{1}$ Department of Nuclear Engineering, Hanyang University, Seoul, Korea \\ ${ }^{2}$ Korea Institute of Nuclear Safety, Daejon, Korea
}

\begin{abstract}
SMART (System-integrated Modular Advanced Reactor) is a small and medium size integrated reactor with $330 \mathrm{MW}_{\text {th }}$ power which has been developed in Korea. In the SMART, some of the components such as steam generator, flow mixing header assembly and flow skirt are installed inside the reactor vessel and near the core, which lead to their activation. The components will be withdrawn for maintenance, inspection, replacement or decommissioning, which causes radiation fields from them. In order to evaluate their activities and radiation fields, a computer-based simulation was performed using the MCNPX code. We calculated the dose rates by distance from the surface of the components to $5 \mathrm{~m}$ away and the variation of dose rates over time. Dose rates near the withdrawn components are extremely high and the dose rate is still extremely high after sufficient cooling time. Thus, approach to these components must be prohibited and all of works relevant with the withdrawn components must be performed by remote control system.
\end{abstract}

\section{KEYWORDS: activation, dose rate, internal components, MCNPX, SMART}

\section{Introduction}

The activation of components inside the reactor vessel is evaluated in order to provide information on radiological environment during maintenance and decommissioning ${ }^{1)}$. Based on this information, maintenance, inspection, replacement or decommissioning technologies are determined to prohibit excessive exposure of workers.

SMART (System-integrated Modular Advanced ReacTor) is a small and medium size system integrated reactor with $330 \mathrm{MW}_{\text {th }}$ power developed in Korea. In the SMART, some of the components such as steam generators, a flow mixing header assembly (FMHA) and a flow skirt are installed inside a reactor vessel and near the core, which is the primary difference between SMART and typical PWRs. Thus, these components are irradiated by fission neutrons, thereby causing activation of these components.

These components can be withdrawn respectively for maintenance or replacement, which makes radiation field near them. Thus, workers can be exposed by radiation while they inspect or maintain these components.

For this reason, it is compulsory to assess the radiation field when the components are withdrawn. In addition, it is also important to estimate the variation in dose rates near the components in order to determine when workers can approach to the components.

In order to assess the dose rate, activity due to the activation of the components was calculated using the MCNPX $\operatorname{code}^{2)}$. Then, the dose rates were estimated using calculated activities with the MCNPX code.

*Corresponding Author, E-mail: jakilee@hanyang.ac.kr

(C) 2012 Atomic Energy Society of Japan, All Rights Reserved.

\section{Methods}

\section{Activity Calculation}

In order to estimate activities of the internal components, reaction rates of activation that occurred in the components were calculated using the MCNPX code.

First, we modeled major structures and the internal components of SMART such as the core, the support barrel, the reactor vessel, steam generators, the FMHA, and the flow skirt using the MCNPX code (Figure 1). Because some of the structures and components such as steam generator tubes have very complex geometry and the MCNPX code has a geometric limit, the shapes of the structures and components were simplified as shown in Figure 1.

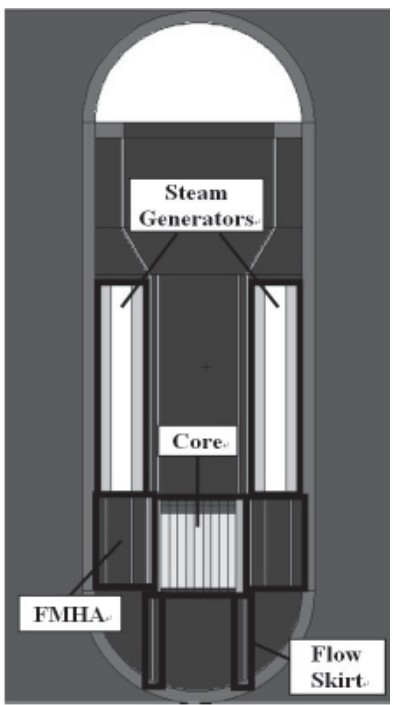

Fig. 1 The modeling of SMART using MCNPX and the major internal components. 
In the next step, we identified the major reactions occurring in the steam generators, the FMHA, and the flow skirt. The FMHA is composed of SS304 stainless steel, and the steam generator tubes and the flow skirt are composed of Inconel 690. The compositions of these materials are shown in Table 1. In addition to these materials, a cobalt impurity of 0.38 weight percent for the SS304 stainless steel ${ }^{3)}$ and that of $180 \mathrm{ppm}$ for Inconel $690^{4)}$ were taken into account. Among reactions which can occur in these materials, we excluded reactions which do not product gamma emitter radioisotopes or long-lived radioisotopes. Through this process we identified major reactions as shown in Table 2.

Table 1 Compositions of the SS304 stainless steel and Inconel 690.

\begin{tabular}{ccc}
\hline \multirow{2}{*}{ Element } & \multicolumn{2}{c}{ Composition (\%) } \\
\cline { 2 - 3 } & SS304 & Inconel 690 \\
\hline $\mathrm{C}$ & 0.08 & - \\
$\mathrm{Si}$ & 0.10 & - \\
$\mathrm{P}$ & 0.05 & - \\
$\mathrm{S}$ & 0.03 & - \\
$\mathrm{Cr}$ & 19.00 & 30.00 \\
$\mathrm{Mn}$ & 2.00 & \\
$\mathrm{Fe}$ & 69.49 & 10.00 \\
$\mathrm{Ni}$ & 9.25 & 60.00 \\
\hline
\end{tabular}

Table 2 Major reactions for activities calculation.

\begin{tabular}{cccc}
\hline Reaction & Product & $\mathrm{T}_{1 / 2}$ & $\begin{array}{c}\text { Gamma energy } \\
(\mathrm{MeV}) \text { and intensity }\end{array}$ \\
\hline${ }^{58} \mathrm{Ni}(\mathrm{n} . \mathrm{p}){ }^{58} \mathrm{Co}$ & ${ }^{58} \mathrm{Co}$ & $70.86 \mathrm{~d}$ & $0.811-99.45 \%$ \\
${ }^{50} \mathrm{Cr}(\mathrm{n}, \gamma){ }^{51} \mathrm{Cr}$ & ${ }^{51} \mathrm{Cr}$ & $27.70 \mathrm{~d}$ & $0.320-9.92 \%$ \\
${ }^{54} \mathrm{Fe}(\mathrm{n}, \mathrm{p}){ }^{54} \mathrm{Mn}$ & ${ }^{54} \mathrm{Mn}$ & $312.12 \mathrm{~d}$ & $0.835-100 \%$ \\
${ }^{58} \mathrm{Fe}(\mathrm{n}, \gamma){ }^{59} \mathrm{Fe}$ & ${ }^{59} \mathrm{Fe}$ & $44.50 \mathrm{~d}$ & $1.099-56.50 \%$ \\
& & & $1.292-43.20 \%$ \\
${ }^{59} \mathrm{Co}(\mathrm{n}, \gamma){ }^{60} \mathrm{Co}$ & ${ }^{60} \mathrm{Co}$ & $5.27 \mathrm{y}$ & $1.173-99.85 \%$ \\
& & & $1.332-99.98 \%$ \\
\hline
\end{tabular}

$\mathrm{T}_{1 / 2}$ : Half-life

In the MCNPX code, the reaction rates are calculated with multiplying neutron flux by atomic density and a corresponding cross section. Because reaction rates in the components vary with distance from the core, the components were equally segmented into three calculation regions with axial direction.

Using the calculated reaction rates, the time-dependent activities in the components were calculated by the equation

$$
A(t)=V R\left(1-e^{-\lambda t}\right)(B q)
$$

where $\mathrm{V}$ is the volume of the component, $\mathrm{R}$ is the reaction rate, $\lambda$ is a decay constant, and $t$ is the irradiation time.

\section{Dose Rate Calculation}

After the activity calculation was completed, we calculated the dose rate from withdrawal of the components. First, we modeled the steam generator, the FMHA, and the flow skirt using MCNPX code when they are withdrawn. In the next step, the calculated activities for each product, and corresponding gamma energy and intensity were used as source terms for the dose rate calculation. In order to calculate the maximum dose rate, the activities of all products were assumed to be saturated.

In order to assess the variation of the dose rate with distance, we divided the distance from the surface of the components to $5 \mathrm{~m}$ away into 25 regions. To calculate the dose rate at each region, we used the mesh based tally which is a function included in the MCNPX code and by which gamma flux can be calculated at each mesh. Using this calculated gamma flux, the dose rate is calculated by multiplying the flux-to-dose conversion factors from ICRP $74^{5)}$.

In addition, we calculated the change of dose rates over time at $1 \mathrm{~m}$ away from the components in order to estimate when workers can approach to the components for maintenance or replacement.

\section{Results and Discussion}

Results show the time-dependent specific activities in the steam generator, the FMHA, and the flow skirt during operation of 20 years and the dose rates near these components when these components are withdrawn. The relative errors of all results using the MCNPX code were below $10 \%$.

\section{Steam Generators}

As mentioned in the section II, the steam generators were segmented into three regions for activities calculation. However, the activities in the middle and upper regions were $10^{3}-10^{5}$ times smaller than those in the lower region. Thus, it was assumed that the activities in the middle and upper regions are negligible for efficient calculation. Figure 2 shows time-dependent activities of five products shown in Table 2 in the lower region of the steam generator.

As shown in Figure 2, ${ }^{51} \mathrm{Cr}$ and ${ }^{60} \mathrm{Co}$ are dominant products in the steam generator during the operation. Even though the steam generator contains much more ${ }^{58} \mathrm{Ni}$ than other nuclides, the amount of ${ }^{58} \mathrm{Co}$ generated by the $(n, p)$ reaction of ${ }^{58} \mathrm{Ni}$ was much less than other products except ${ }^{54} \mathrm{Mn}$. This is because the energy of fission neutrons decreases while they travel from the core to the steam generator. Thus, the reaction rate of the $(n, p)$ reaction decreases because the $(n, p)$ reaction has a threshold of a few $\mathrm{MeV}$ and the cross section of $(\mathrm{n}, \mathrm{p})$ reaction for low energy neutron is much less than that for high energy neutron.

\section{FMHA}

As mentioned in the section II, the FMHA was segmented into three regions for activities calculation. However, the activities in the outer region which is the farthest from the core were $10^{5}-10^{6}$ times smaller than those in the inner and middle regions. Thus, it was assumed that the activities in the outer regions are negligible for efficient calculation. Figure 3 shows time-dependent total activities of five products shown in Table 2 in the inner and middle regions of the FMHA.

Similar to the activities in the steam generator, ${ }^{51} \mathrm{Cr}$ and ${ }^{60} \mathrm{Co}$ are dominant products in the steam generator during the operation. However, the activities in the FMHA are about 
$10^{4}$ times higher than those in the steam generator. This is because the FMHA is installed much closer to the core than steam generator.

In addition, ${ }^{60} \mathrm{Co}$ becomes the most dominant isotope after 5 year of operating time in the FMHA while ${ }^{51} \mathrm{Cr}$ is the most dominant during all the operating times in the steam generator. This is because SS304, the material of the FMHA, has more cobalt impurity than Inconel690, material of the steam generator tubes.

\section{Flow Skirt}

As mentioned in the section II, the flow skirt was segmented into three regions for activities calculation. However, the activities in the middle and lower regions were $10^{3}-10^{5}$ times smaller than those in the lower region. Thus, it was assumed that the activities in the middle and lower regions are negligible for efficient calculation. Figure 4 shows time-dependent activities of five products shown in Table 2 in the lower region of the flow skirt.

The activities in the flow skirt have similar tendency with those in the steam generator because the flow skirt is also composed of Inconel690 like the steam generator tubes. However, the activities in the flow skirt are about $10^{2}$ times higher than those in the steam generator

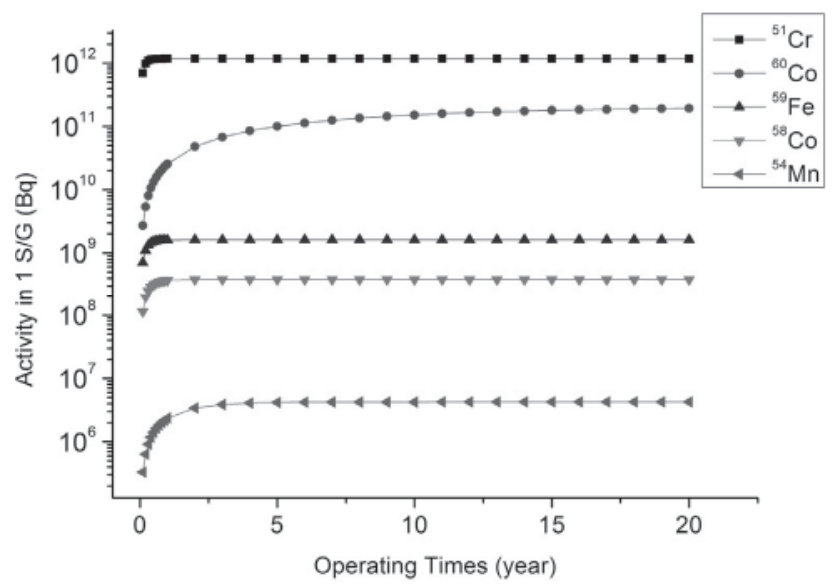

Fig. 2 Activity during operation of 20 years in the steam generator.

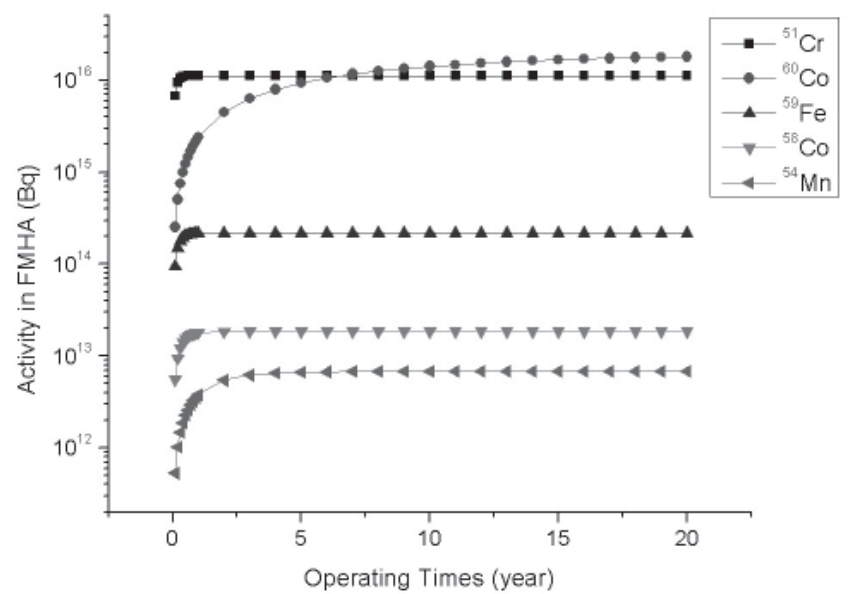

Fig. 3 Activity during operation of 20 years in the FMHA.

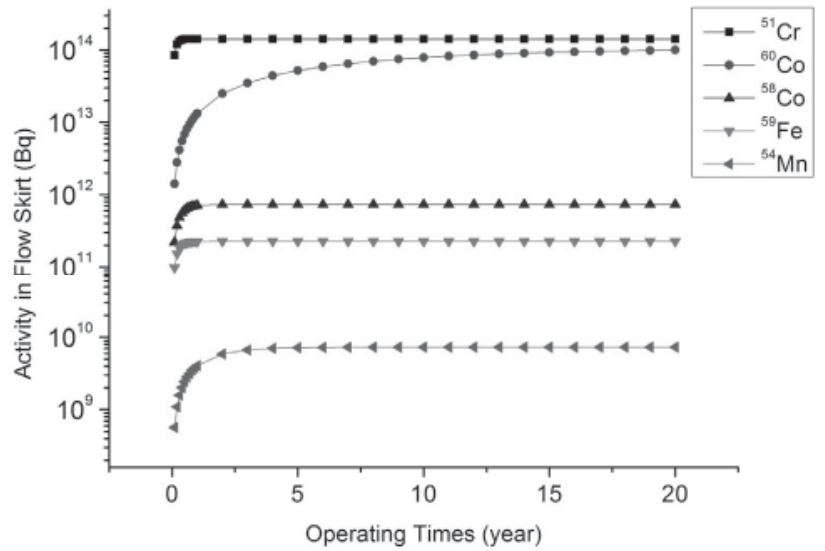

Fig. 4 Activity during operation of 20 years in the flow skirt.

\section{Dose Rate}

Figure 5 shows the dose rate near the withdrawn components by distance from the surface of them. As shown in Figure 5, the dose rate near the FMHA and the flow skirt exceeds $10 \mathrm{~Sv} / \mathrm{h}$ which is extremely high dose rate. Even if the dose rate near the steam generator is relatively lower than other components, the dose rate exceeding $10 \mathrm{mSv} / \mathrm{h}$ is also very high dose rate. Thus, approach to these components must be prohibited to prevent excessive exposure for workers when they are withdrawn and the withdrawn components must be stored in a water pool.

Figure 6 shows the change of the dose rate over cooling time at the $1 \mathrm{~m}$ away from the component. As shown in Figure 6, the dose rate near the components decreases gently over cooling time because ${ }^{60} \mathrm{Co}$ which has long half-life and emits high energy gamma rays, thereby largely contributing the dose rate, is the dominant radioisotope in the components. Thus, even if the components are cooled during sufficient time, all of works such as maintenance, inspection, replacement and decommissioning must be performed by remote control system.

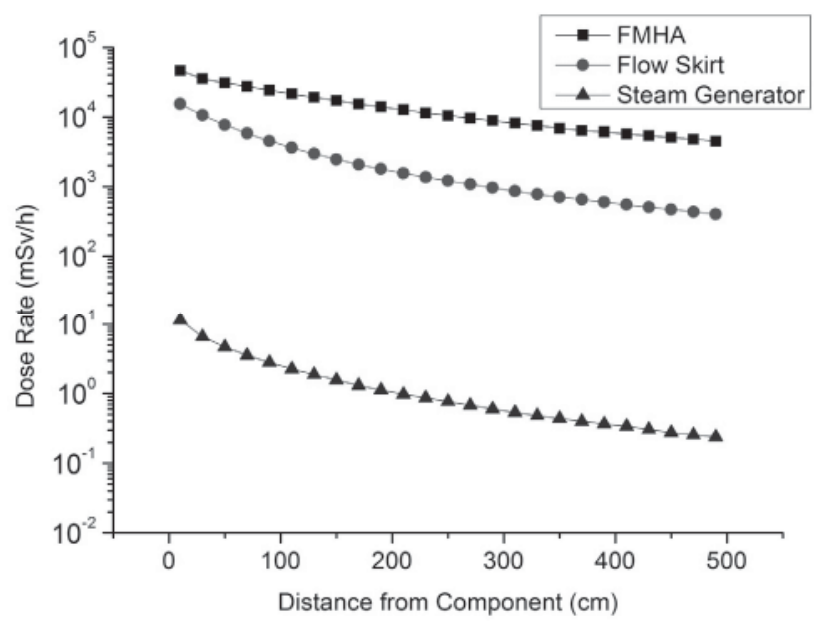

Fig. 5 The dose rate near the withdrawn components by distance. 


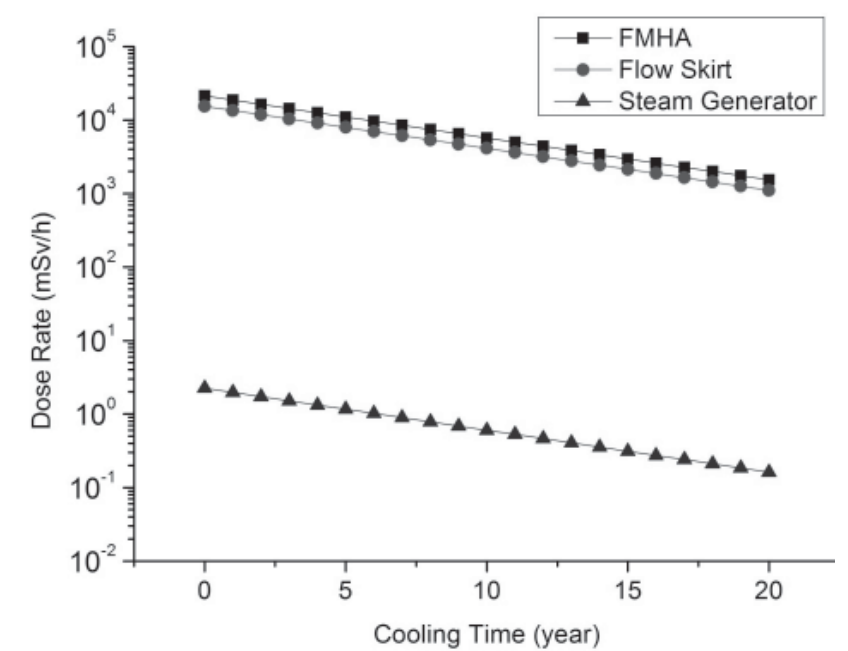

Fig. 6 The change of dose rate over cooling time.

\section{Conclusion}

We calculated the activities due to the activation of the components and the dose rates near the withdrawn components. In order to estimate the activities, the reaction rates were calculated using the MCNPX code. Using these activities, the dose rate near the components and the variation of the dose rate over time were evaluated.

Because the neutron energy decreases as the fission neutrons travel from the core to the components, the $(n, p)$ reaction rates having high energy thresholds also decrease. Thus, ${ }^{51} \mathrm{Cr}$ and ${ }^{60} \mathrm{Co}$ from the $(\mathrm{n}, \gamma)$ reactions become the dominant products in the components. The dose rates near the components were extremely high, which indicates that approach to these components must be prohibited to prevent excessive exposure for workers and these components must be stored in a water pool. In addition, even if the components are cooled during sufficient time, all of works such as maintenance, inspection, replacement and decommissioning must be performed by remote control system because the dose rate after sufficient cooling time is still extremely high.

\section{Acknowledgment}

This research was supported by the Development Program for Nuclear Technology (No. 20110039) of Korean Ministry of Education, Science and Technology.

\section{References}

1) Kyo-Youn Kim et al, "Preliminary Estimation of Long-lived Activation Products in the Reactor Structures of SMART, Progress in NUCLEAR SCIENCE and TECHNOLOGY, Vol. 1, (2011)

2) Denise B. Pelowitz, "MCNPX user's manual, Version 2.5.0", Los Alamos National Laboratory, (2005).

3) A. S. Zhilkin, O. V. Starkov and E. V. Shestopalov, Cobalt and other impurities in reactor Steels, Atomnaya Énergiya, 52[4], 261(1982).

4) Karl-Heinz Neeb, The Radiochemistry of Nuclear Power Plants with Light Water Reactors, Walter de Gruyter \& Co., (1997).

5) ICRP, Conversion coefficients for use in radiological protection against external radiation, The International Commission on Radiological Protection publication 74. Ann. ICRP 26(3/4) (1996) 19 